\title{
Exploratory and Confirmatory Bayesian Networks Identify the Central Role of Non-judging in Symptoms of Depression
}

\author{
Mikael Rubin ${ }^{1}$ (1) $\cdot$ Santiago Papini ${ }^{1} \cdot$ Justin Dainer-Best ${ }^{2} \cdot$ Eric D. Zaizar $^{1} \cdot$ Jasper A. J. Smits $^{1} \cdot$ Michael J. Telch $^{1}$
}

Accepted: 7 August 2021 / Published online: 19 August 2021

(c) The Author(s), under exclusive licence to Springer Science+Business Media, LLC, part of Springer Nature 2021

\begin{abstract}
Objectives Depression is a highly heterogeneous disorder, and meta-analyses of mindfulness-based interventions show moderate efficacy for reducing depressive symptoms. However, the mechanisms governing their efficacy remain unclear, highlighting the need for hypothesis-generating analyses to guide future research.

Methods We used Bayesian network analysis in three cross-sectional samples $(N=1135)$ of undergraduates and participants from the community to identify links between individual symptoms of depression and specific facets of mindfulness. In two exploratory studies, we assessed depression using the Patient Health Questionnaire $(n=384)$ or the Depression Anxiety and Stress Scale $(n=350)$ and mindfulness using the Five-Facet Mindfulness Scale.

Results Across these samples and measures, exploratory analyses indicated that non-judging was a central bridge between facets of mindfulness and symptoms of depression. We confirmed this finding in a pre-registered replication $(n=401)$ using a recently developed confirmatory testing framework for network analysis. Non-judging was consistently a central bridge in the networks and specifically linked to the symptoms of depression related to feelings of failure and worthlessness.

Conclusions These findings provide strong evidence that non-judging is an essential feature of mindfulness in the context of depression and provides direction for future research testing mindfulness-oriented treatment prescriptions for depression.
\end{abstract}

Keywords Mindfulness · Depression · Gaussian graphical model $\cdot$ Bayesian network analysis

Depression is a heterogeneous psychological concern (Zimmerman et al., 2015), with symptom profiles differing widely between individuals, suggesting that uniform treatment approaches may be misguided (Fried \& Nesse, 2015). Meta-analyses of specific, manualized mindfulness based interventions (MBIs) such as mindfulness-based cognitive therapy and mindfulness-based stress reduction have shown moderate reductions in symptoms of depression (Chi et al., 2018; Galante et al., 2013). The relationship between dispositional mindfulness and depression has been relatively well established, with greater self-reported mindfulness associated with fewer symptoms of depression in both clinical (Gu et al., 2016) and non-clinical (Medvedev et al., 2018) samples. Moreover, mindfulness training leads to greater

Mikael Rubin

mikaelrubin@utexas.edu

1 Department of Psychology and Institute for Mental Health Research, The University of Texas At Austin, 108 E. Dean Keeton, A8000, Austin, TX 78712, USA

2 Department of Psychology, Bard College, NY, USA dispositional mindfulness (Kiken et al., 2015). Mindfulness is considered a multi-dimensional construct (Baer, 2011), and the relationship between different facets of mindfulness and symptoms of depression varies (Medvedev et al., 2018). Thus, identifying specific facets of mindfulness associated with depression may provide guidance for future research to test the role of specific mindfulness-based targets in intervention-based research.

Network analysis - an item-level approach that evaluates individual features of a construct instead of their aggregate (i.e., mean level) has grown in popularity and has become an important tool for psychopathology research, including depression (Bringmann et al., 2015). For example, using network analyses, Medvedev et al. (2021) found a strong inverse association between the mindfulness facet of nonjudgment and emotional distress; however, specific symptoms of depression were not examined. There is significant value in addressing individual symptoms of depression because of the wide range of different symptom profiles for depression, where heterogeneity among individuals can lead to misleading conclusions about the interrelationships 
among constructs (Fried \& Nesse, 2015). Conceptualizing mindfulness from the network perspective is somewhat different as it does not reflect symptoms, but rather a complex set of intersecting forms of present-moment attention. Importantly, there is evidence that mindfulness can be measured through self-report as a trait (Truong et al., 2020), rather than reflecting only the state of mindfulness. The network perspective can also provide insight into complex interrelations between different features of mindfulness cross-sectionally (Heeren et al., 2021) as well as before and after an intervention (mindfulness-based stress reduction; Roca et al., 2019). Taken together emerging research suggests that using network analysis to investigate the relationship between facets of mindfulness and specific symptoms of depression is of interest in better understanding how to develop targeted hypotheses that can then be tested in clinical intervention research.

The primary aim of this study was to identify associations between specific symptoms of depression and facets of mindfulness. The second aim of the study was to test the robustness of associations between symptoms of depression and facets of mindfulness. We hypothesized that facets of mindfulness would be negatively associated with symptoms of depression, but made no specific a priori hypotheses for samples 1 and 2 (exploratory). For sample three (pre-registered, confirmatory), we hypothesized that the facets of non-judging and awareness would be negatively associated with symptoms of depression.

\section{Methods}

\section{Participants}

Participants' demographics are included in Table 1. Participants for the studies were (a) undergraduate students who participated in the online surveys for research credit as part of

Table 1 Demographic summary

\begin{tabular}{|c|c|c|c|}
\hline & Sample $1(n=384)$ & Sample $2(n=350)$ & Sample $3(n=401)$ \\
\hline & \multicolumn{3}{|l|}{ Mean (SD) } \\
\hline Age & $22.89(9.92)$ & $18.96(1.40)$ & $24.84(11.06)$ \\
\hline FFMQ-15 total & $45.92(6.87)$ & $41.52(7.71)$ & $46.48(6.99)$ \\
\hline FFMQ-observing & $8.86(2.53)$ & $8.74(2.83)$ & $9.04(2.83)$ \\
\hline FFMQ-describe & $9.06(2.70)$ & $9.09(2.88)$ & $9.24(2.53)$ \\
\hline FFMQ-awareness & $9.50(2.42)$ & $9.96(2.64)$ & $9.86(2.83)$ \\
\hline FFMQ-non-judging & $9.99(2.84)$ & $10.15(3.32)$ & $9.73(3.27)$ \\
\hline FFMQ-non-reactivity & $8.52(2.53)$ & $8.15(2.60)$ & $8.62(2.70)$ \\
\hline PHQ-8 total score & $8.99(6.05)$ & N/A & $9.42(6.37)$ \\
\hline \multirow[t]{2}{*}{ DASS-21 depression subscale } & N/A & $25.13(9.81)$ & $28.56(10.83)$ \\
\hline & \multicolumn{3}{|l|}{ No. $(\%)$} \\
\hline Sex (female) & $238(62.0)$ & $267(76.3)$ & $261(65.1)$ \\
\hline Ethnicity (Hispanic) & $119(31.0)$ & $118(33.7)$ & $133(33.2)$ \\
\hline \multicolumn{4}{|l|}{ Race } \\
\hline Hispanic & $89(23.2)$ & $64(18.3)$ & $69(17.2)$ \\
\hline $\begin{array}{l}\text { Black or African } \\
\text { American }\end{array}$ & $19(4.9)$ & $17(4.9)$ & $25(6.2)$ \\
\hline Asian & $65(16.9)$ & $89(25.4)$ & $62(15.5)$ \\
\hline White & $190(49.5)$ & $133(38.0)$ & $204(50.9)$ \\
\hline Multiple & $44(11.5)$ & $47(13.4)$ & $39(9.7)$ \\
\hline $\begin{array}{l}\text { American Indian or } \\
\text { Alaska Native }\end{array}$ & $0(0)$ & $0(0)$ & $1(.2)$ \\
\hline $\begin{array}{l}\text { Native Hawaiian or } \\
\text { Other Pacific Islander }\end{array}$ & $0(0)$ & $0(0)$ & $1(.2)$ \\
\hline Unknown/not reported & $2(0.5)$ & $0(0)$ & $0(0)$ \\
\hline Sample (community) & $57(14.8)$ & $0(0)$ & $142(35.1)$ \\
\hline Possible depression (PHQ-8 $\geq 10$ ) & $164(42.7)$ & N/A & $178(44.4)$ \\
\hline High depression (DASS subscale $\geq 21$ ) & $N / A$ & $198(56.6)$ & $279(69.8)$ \\
\hline Unemployment began during COVID-19 & $73(19.0)$ & $62(17.7)$ & $49(12.2)$ \\
\hline Knows someone who was infected & $103(26.8)$ & $252(72.0)$ & $299(74.6)$ \\
\hline Knows someone who died & $13(3.3)$ & $52(14.9)$ & 88 (21.9) \\
\hline
\end{tabular}


an introductory course (sample $1=85.2 \%$; sample $2=100 \%$; sample $3=64.9 \%$ ); (b) individuals recruited online by posting on web-boards and social media who participated without compensation (sample $1=14.8 \%$; sample $2=0 \%$; sample $3=0 \%$ ); or (c) participants who were recruited through Amazon Mechanical Turk (MTURK) and were compensated \$1 (sample $1=0 \%$; sample $2=0 \%$; sample $3=35.1 \%$ ). All participants provided written, informed consent, and the Institutional Review Board of The University of Texas at Austin approved all procedures.

\section{Procedures}

Prior to completing the survey, participants signed an informed consent document. Several attention check questions were included in the survey, and participants who answered any of these incorrectly were excluded from further participation. Upon completing the survey, participants were thanked for their time and automatically redirected to receive credit for participation (undergraduate students) or provided with a code used to validate their participation (MTURK workers).

\section{Measures}

\section{Five Facet Mindfulness Questionnaire-15 (FFMQ-15; Baer et al., 2008)}

The FFMQ-15 is a 15-item self-report measure that assesses the five components of mindfulness, including (1) observing; (2) describe; (3) act with awareness; (4) non-judging; and (5) non-reactivity. Participants rated the truth of each statement on a 5-point scale from "never or very rarely true" to "very often or always true". For sample 1, the total alpha coefficient was 0.67 , and the subscale coefficients ranged from 0.81 to 0.56 ; for sample 2 , the total coefficient was 0.69 , and the subscale coefficients ranged from 0.81 to 0.49 ; for sample 3 , the total coefficient was 0.63 , and the subscale coefficients ranged from 0.85 to 0.39 .

\section{Patient Health Questionnaire-8 (PHQ-8; Kroenke et al., 2009)}

The PHQ-8 is an 8-item self-report scale assessing symptoms of depression. Participants rated how often they had been bothered by each concern within the last 2 weeks on a 4-point scale (0-3) from "not at all" to "nearly every day." For sample 1, the alpha coefficient was 0.89 ; for sample 3, the coefficient was 0.90 .
Depression, Anxiety, and Stress Scale (DASS-21; Henry \& Crawford, 2005)

The DASS-21 is a 21-item self-report questionnaire designed to measure the severity of symptoms common to both depression and anxiety. Responders are required to indicate the presence of a symptom over the previous week. Each item is scored from 0 (did not apply to me at all over the last week) to 3 (applied to me very much or most of the time over the past week). For the current study, only the depression subscale was administered. For sample 2, the alpha coefficient was 0.91 ; for sample 3 , the coefficient was 0.92 .

\section{Data Analyses}

$\mathrm{R}$ was used to conduct all analyses. The syntax, data, and the replication preregistration for sample 3 are available at (https://osf.io/hvzrd/).

We conducted the network analysis using a Bayesian Gaussian graphical model (BGGM) estimated using the $B G G M$ package (Williams \& Mulder, 2020). This approach estimates an inverted variance-covariance matrix to represent the relation between any two items. This means that the estimate of each association in the network is controlling for all other associations. In BGGM, relationships between items reflect the posterior means of the associations. This is distinct from the other Bayesian approach taken in the network analysis literature - directed acyclic graphs (DAGs) because DAGs constrain the association between nodes to be unidirectional. There are challenges associated with interpreting cross-sectional data causally, especially when the associations are unidirectional (Epskamp et al., 2018), and we chose the exploratory/confirmatory process facilitated by the BGGM package to strengthen our interpretations of cross-sectional network models.

For the exploratory analyses, the "explore" function in BGGM with specified "exhaustive" selection was used, which provides a network of positive associations and negative associations using a Bayes factor cutoff of 3 (moderate support) to determine which associations to include. For the confirmatory analyses, the "confirm" function was used which tests equality constraints of the posterior probability of a given hypothesis (which were all pre-registered) and which provides a Bayes factor value estimating the relative support for which hypothesis the results fall under (i.e., null or alternative). Bayes factors have been conceptualized as reflecting the strength of evidence in favor of the alternative hypothesis or null hypothesis, where 1 reflects no evidence, 3 reflects anecdotal evidence in favor of the alternative hypothesis, and 1/3 reflects anecdotal evidence in favor of the null hypothesis, with greater evidence for the alternative or null as values grow or approach zero, respectively (Wetzels \& 
Wagenmakers, 2012). Bayes factors can be nearly infinitely large or approach zero, and the values reflect only the confidence of the estimate - not the strength of the estimate itself. We scaled and centered all variables and used the weighted topological overlap approach from EGAnet (Golino et al., 2020) to aggregate the mindfulness facets into latent variable scores. Finally, we evaluated bridge centrality using the networktools package. Bridge nodes are variables that are associated within a specific measure and across distinct measures. Bridge node centrality is a measure of the total strength of the associations of a specific variable with variables in a different community (essentially a sum of the correlation coefficients that are bridging between communities). For the confirmatory analyses, we used the "confirm" function in BGGM with the default prior (0.25) and specified the hypotheses that we preregistered a priori.

Additionally, we conducted pre-registered exploratory analyses to test differences between the networks. We used the ggm_compare_estimate function to compute differences between each association in each network (comparing the networks of samples 1 and 3 on the PHQ and the FFMQ and samples 2 and 3 on the DASS depression subscale and the FFMQ).

\section{Results}

\section{Sample 1 Exploratory Network Findings}

Fig. 1 A provides a graphical representation of the exploratory network analyses from the first sample. The non-judging facet had a bridge centrality of 0.339 , and the awareness facet had a bridge centrality of 0.313 , with negative associations to symptoms of depression. Unexpectedly, we also found that the describe facet had a bridge centrality of 0.143 with a positive association with a "Poor appetite or overeating?" Whereas negative associations suggest that greater mindfulness is associated with lower symptoms of depression (as is commonly reported in the literature), a positive association indicates the opposite

\section{Sample 2 Exploratory Network Findings}

Fig. $1 \mathrm{~B}$ provides a graphical representation of the exploratory network analyses from the second sample. Non-judging was the only bridge between mindfulness and the symptoms of depression "I felt I wasn't worth much as a person" and "I found it difficult to work up the initiative to do things" (0.289). While awareness was linked to the symptom of depression "I found it difficult to work up the initiative to do things", the facet was not found to be central to the network.

\section{Sample 3 Pre-registered Network Findings}

Fig. $1 \mathrm{C}$ and $\mathrm{D}$ provide a graphical representation of the third sample's confirmatory network analyses. We tested the specific hypotheses generated in the exploratory stages of the analysis, which confirmed our pre-registered primary hypotheses that non-judging would bridge mindfulness and symptoms of depression. However, the evidence was much stronger for the centrality of non-judging when using the PHQ-8 to assess depression (Bayes Factor $(B F)=7,998.671)$ than when using the DASS-21 depression subscale $(B F=2.448)$. We also confirmed the centrality of the awareness facet of mindfulness in the PHQ- 8 network $(\mathrm{BF}=9.915)$

The pre-registered exploratory comparisons between the networks showed that the strengths of individual associations were not meaningfully different in the vast majority of cases (Supplementary Figures 1 and 2), suggesting that the associations within the networks were likely to be similar. In comparing sample 1 and sample 3 , there were no meaningful differences in edge strength between non-judging and symptoms of depression measured by the PHQ-8; of the 78 total edges of which only $6(8 \%)$ were different. In comparing samples 2 and 3, there were no meaningful differences in edge strength between non-judging and the DASS-21 depression subscale; of the 66 total associations in the network, only $2(3 \%)$ had meaningfully different associations.

We found only anecdotal evidence to support our secondary hypothesis that the association between "Poor appetite or overeating?" and the describe facet was null $(\mathrm{BF}=0.447)$. There was inconclusive evidence for the secondary hypothesis that the symptom of "Feeling bad about yourself" on the PHQ-8 was more strongly associated with non-judging than "Feeling down, depressed, or hopeless" $(\mathrm{BF}=1.033)$, and for the secondary hypothesis that the symptom of "I felt I wasn't worth much as a person" on the DASS-21 depression subscale was more strongly associated with non-judging than the symptom of "I found it difficult to work up the initiative to do things" $(\mathrm{BF}=1.357)$.

\section{Discussion}

From two exploratory analyses, we developed the dataderived hypothesis that non-judging serves as the most central bridge between mindfulness and depression when controlling for all other associations. We then preregistered this primary hypothesis (along with several secondary ones), collected new data, and confirmed this hypothesis in a subsequent network analysis. However, there was a large degree of difference in the confidence of the confirmatory analysis depending on the measure of depression. While the PHQ-8 showed robust evidence in favor of the hypothesis, 
A

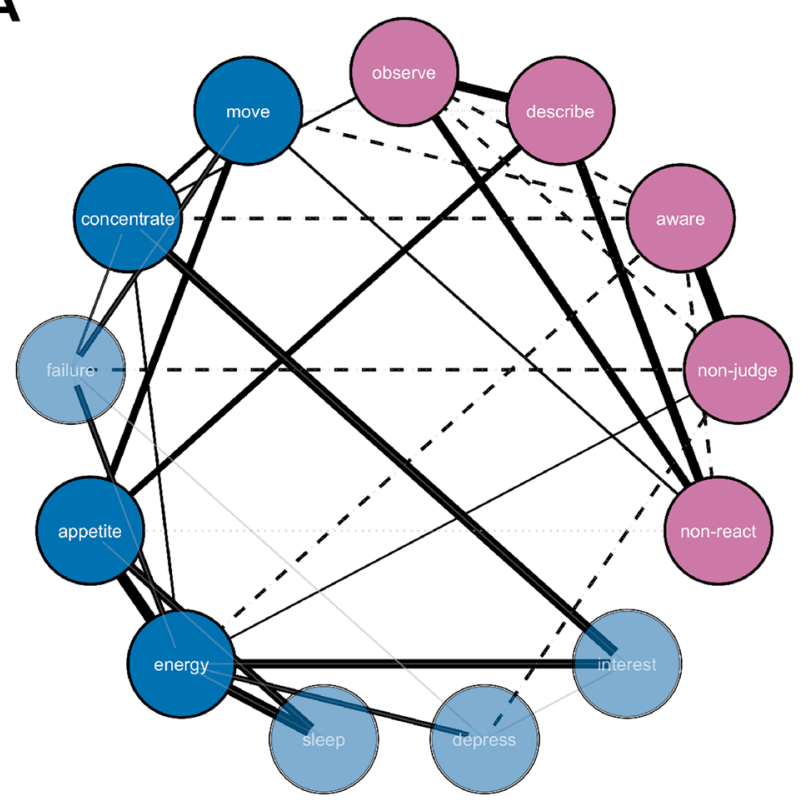

C

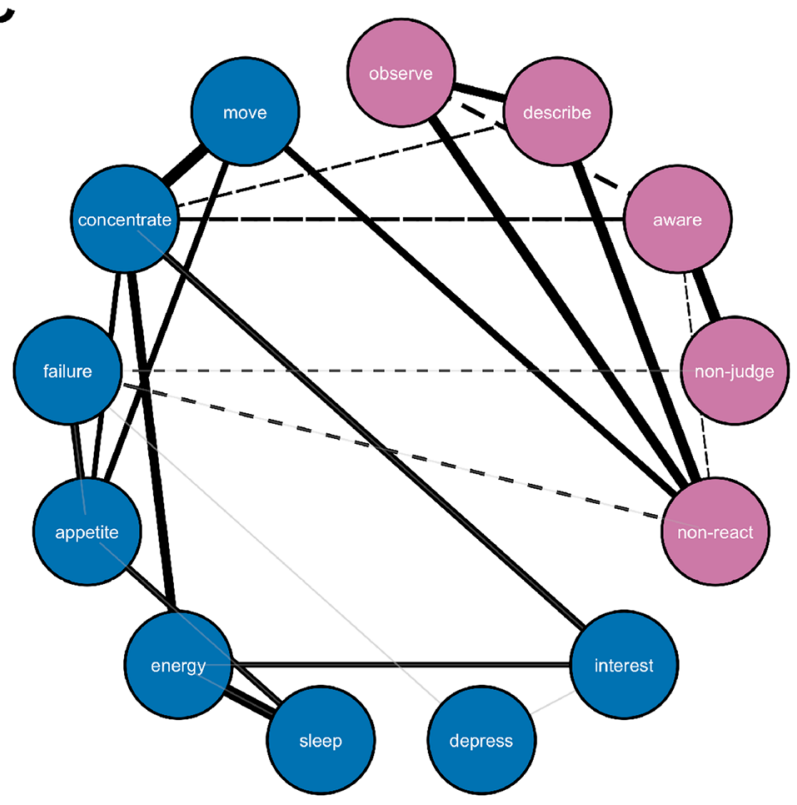

Depression Symptoms

Fig. 1 Gaussian graphical models. A Exploratory network PHQ and FFMQ. B Exploratory network DASS and FFMQ, bridge nodes are highlighted. C Confirmatory network PHQ and FFMQ. D Confirmatory network DASS and FFMQ. Pink (lighter) nodes are mindfulness facets (Five Facet Mindfulness Questionnaire-FFMQ), Blue (darker) nodes are depression facets (in A, C: Patient Health Questionnaire-PHQ; in B, D: Depression Anxiety and Stress Scale-
B

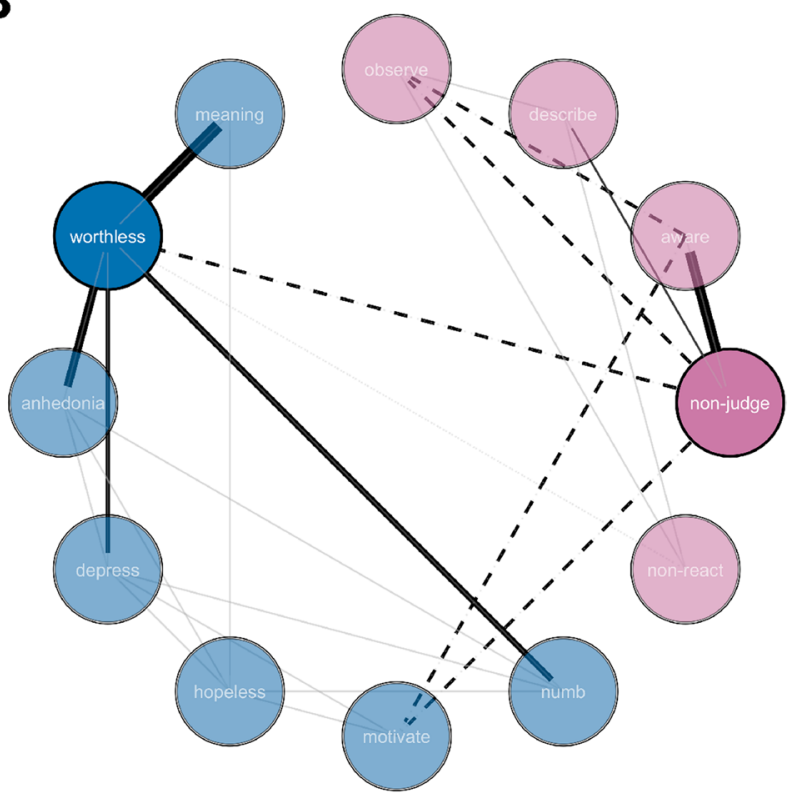

D

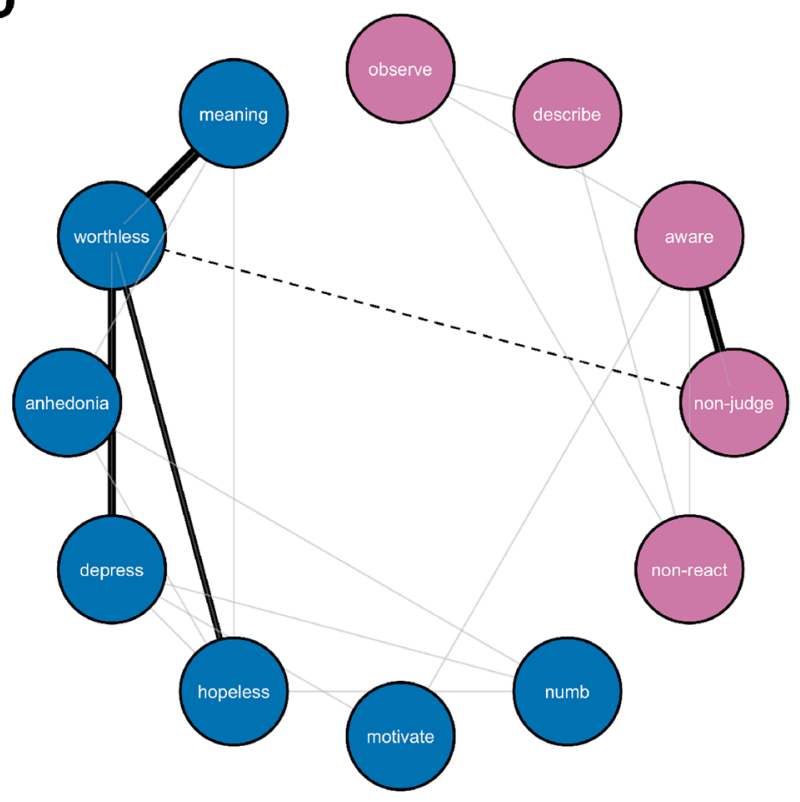

Mindfulness Facets (FFMQ)

DASS, depression subscale). In $\mathbf{A}$ and $\mathbf{B}$, only the central nodes are filled; if a node was not central, it was faded. Solid lines reflect positive associations; dashed lines reflect negative associations. Grayed lines reflect associations without sufficient evidence to support either the null or alternative hypotheses. The width of the lines reflects the strengths of the associations 
the evidence supporting the bridge centrality of non-judging when assessing depression using the DASS-21 subscale was much weaker. We did demonstrate that in comparing association strengths between networks, individual associations remained similar. However, there was substantial variability in association strengths such that the evidence to support specific differences in magnitude between associations was inconclusive.

This variability between samples highlights a critical element of the approach we took - namely, that consideration of the specific measure used may influence specific interrelations among facets of mindfulness and specific symptoms of depression. Moreover, comparing mindfulness facets and symptoms on the PHQ-8, there was insufficient evidence to confirm the order of the strength of specific associations between non-judging and symptoms of depression. One potential interpretation may be that the PHQ- 8 and DASS21 depression subscale measure depression in somewhat different ways. The PHQ was specifically developed as a brief screening assessment tool for depression (Kroenke et al., 2009), whereas the DASS was developed as an integrated measure of negative affect, although the measurement of depression within the scale is distinct from the other scales (Henry \& Crawford, 2005). While highly correlated in sample 3 ( $r=0.783,95 \%$ CI $[0.742,0.818]$ ), symptoms of depression are phrased quite differently between the two scales. For instance, depressed feelings in the PHQ are "Feeling down, depressed, or hopeless?" whereas in the DASS, they are "I felt down-hearted and blue" with a separate question for hopelessness ("I felt that I had nothing to look forward to"). Moreover, the PHQ includes some symptoms of depression that the DASS does not (e.g., appetite and concentration); in particular, the presence/absence of a concentration symptom may explain why the awareness facet was only central for the PHQ-8 network. Prior work has highlighted the heterogeneity of depression symptoms (Fried $\&$ Nesse, 2015). In cross-sectional networks, it may be tempting to draw conclusions about the strength of specific symptom associations. However, our findings suggest that caution is needed when interpreting specific associations in networks and when interpreting differences in the magnitude of associations within a single cross-sectional network. It is worth noting that we identified few differences in comparing the networks, suggesting that associations remained relatively stable even across cross-sectional samples (as has been found by other researchers, for instance in the context of post-traumatic stress disorder, Duek et al., 2021).

Self-criticism has long been considered a central feature of depression (Beck, 1964). In the context of mindfulness, the facet of non-judging has been shown to mediate the relationship between self-criticism and depression (Rohde et al., 2014). Consistent with another recent network analysis (Medvedev et al., 2021), our findings underscore the potential importance of non-judging in the context of depression. While promoting non-judging is a core feature of MBIs, there is little existing work testing specific mechanisms of change within MBIs for depression. Research investigating changes in mindfulness during MBIs for depression, generally shows moderate increases in mindfulness across all facets (Gu et al., 2016; Takahashi et al., 2019). Yet, not all individuals respond to MBIs equally. Thus, our results suggest that particular consideration of individuals experiencing depression that are low in non-judging is warranted. One potential test in future research is whether emphasizing compassion-based practices among those low in non-judging may serve to enhance MBIs for depression. Our results suggest this might be linked to when individuals present with specific symptom profiles of depression (e.g., worthlessness in the DASS or feelings of failure in the PHQ), although future research is needed to confirm this hypothesis.

\section{Limitations and Future Research}

The study has several limitations, including the moderate sample size and that the majority of participants were undergraduate students. However, symptom severity across samples was relatively high, and our confirmatory sample included a large proportion of community participants. Additionally, the internal reliability of some of the mindfulness subscales was fairly low, which may have impacted our findings. It is also the case that mindfulness items can be interpreted differently by meditators and non-meditators, and our lack of information regarding meditation experience in our samples is a limitation that should be considered in future research. Our pre-registered confirmatory Bayesian network approach addresses some but not all the limitations of network analysis. The Bayesian network approach does not remedy psychometric inadequacies such as the low internal reliability of the observe facet nor does it take into account the construct validity of the measures (Epskamp, et al., 2017a, 2017b; Hallquist et al., 2019). Further research on the relationship between mindfulness and depression using novel-integrated methods such as latent network models (Epskamp, et al., 2017a, 2017b) is warranted. Additionally, in sample 3, participants completed two different measures of depression within the same survey, which may lead to a "common methods bias" where responses to one questionnaire influenced responses to the other questionnaire (Podsakoff et al., 2012). Also, a large proportion of sample 3 data (35\%) was collected from MTURK. While we used attention checks and an open-ended response item to reduce the incidence of "bots," automated, or inattentive responding, there is growing concern that the quality of MTURK data has become increasingly poor since 2018 (Chmielewski \& Kucker, 2020), which limits the conclusions we can draw. Of further note, these data were collected during the 
COVID-19 pandemic. It is possible that there were meaningful influences in COVID-19-related experiences (such as a loss due to COVID-19) that may have impacted the network structure. While we did not address the influence of the pandemic within the analyses, we do summarize some relevant data in Table 1.

The current investigation identified and confirm the bridge centrality of non-judging for symptoms of depression across two distinct measures of depression. These findings provide further evidence for the importance of non-judging in the context of depression as well as links to specific symptoms reflecting heightened self-criticism (e.g., feelings of failure or worthlessness). Our findings also underscore how different measures of depression may influence the conclusions drawn in relation to mindfulness. Future research should consider testing whether low non-judging is implicated in poorer treatment outcomes of MBIs for depression. Targeting non-judging, perhaps by emphasizing compassion-based practices, may enhance treatment outcomes for depression.

Supplementary Information The online version contains supplementary material available at https://doi.org/10.1007/s12671-021-01726-1.

Author Contribution MR: designed and executed the study, conducted the data analyses, and wrote the first draft of the paper. SP, JDB: collaborated with the design and writing of the study. JDB: conducted the data visualization. MJT: provided supervision of the study. MJT, EZ, JAJS: collaborated in the writing and editing of the final manuscript.

Data Availability All data and syntax are available at the Open Science Framework (https://osf.io/hvzrd/).

\section{Declarations}

Ethics Approval The study received institutional review board approval from the University of Texas at Austin IRB and was conducted in accordance with relevant national and international ethical standards. Informed consent was obtained from all study participants.

Conflict of Interest The authors declare no competing interests.

\section{References}

Baer, R. A., Smith, G. T., Lykins, E., Button, D., Krietemeyer, J., Sauer, S., \& Williams, J. M. G. (2008). Construct validity of the five facet mindfulness questionnaire in meditating and nonmeditating samples. Assessment, 15(3), 329-342. https://doi.org/10.1177/ 1073191107313003

Baer, R. A. (2011). Measuring mindfulness. Contemporary Buddhism, 12(1), 241-261. https://doi.org/10.1080/14639947.2011.564842

Beck, A. T. (1964). Thinking and depression: II. Theory and therapy. Archives of General Psychiatry, 10(6), 561-571. https://doi.org/ 10.1001/archpsyc.1964.01720240015003

Bringmann, L. F., Lemmens, L. H. J. M., Huibers, M. J. H., Borsboom, D., \& Tuerlinckx, F. J. P. M. (2015). Revealing the dynamic network structure of the Beck Depression Inventory-II. Psychological Medicine, 45(4), 747-757. https://doi.org/10.1017/S0033 291714001809

Chi, X., Bo, A., Liu, T., Zhang, P., \& Chi, I. (2018). Effects of mindfulness-based stress reduction on depression in adolescents and young adults: A systematic review and meta-analysis. Frontiers in Psychology, 9, 1034. https://doi.org/10.3389/fpsyg.2018. 01034

Chmielewski, M., \& Kucker, S. C. (2020). An MTurk crisis? Shifts in data quality and the impact on study results. Social Psychological and Personality Science, 11(4), 464-473. https://doi.org/10. 1177/1948550619875149

Duek, O., Spiller, T. R., Pietrzak, R. H., Fried, E. I., \& Harpaz-Rotem, I. (2021). Network analysis of PTSD and depressive symptoms in 158,139 treatment-seeking veterans with PTSD. Depression and Anxiety, 38(5), 554-562. https://doi.org/10.1002/da.23112

Epskamp, S., Kruis, J., \& Marsman, M. (2017a). Estimating psychopathological networks: Be careful what you wish for. PLOS ONE, 12(6), e0179891. https://doi.org/10.1371/journal.pone.0179891

Epskamp, S., Rhemtulla, M., \& Borsboom, D. (2017b). Generalized network psychometrics: Combining network and latent variable models. Psychometrika, 82(4), 904-927. https://doi.org/10.1007/ s11336-017-9557-x

Epskamp, S., Waldorp, L. J., Mõttus, R., \& Borsboom, D. (2018). The Gaussian graphical model in cross-sectional and time-series data. Multivariate Behavioral Research, 53(4), 453-480. https://doi. org/10.1080/00273171.2018.1454823

Fried, E. I., \& Nesse, R. M. (2015). Depression sum-scores don't add up: Why analyzing specific depression symptoms is essential. BMC Medicine, 13(1), 1-11. https://doi.org/10.1186/ s12916-015-0325-4

Galante, J., Iribarren, S. J., \& Pearce, P. F. (2013). Effects of mindfulness-based cognitive therapy on mental disorders: A systematic review and meta-analysis of randomised controlled trials. Journal of Research in Nursing, 18(2), 133-155. https://doi.org/10.1177/ 1744987112466087

Golino, H., Shi, D., Christensen, A. P., Garrido, L. E., Nieto, M. D., Sadana, R., ... \& Martinez-Molina, A. (2020). Investigating the performance of exploratory graph analysis and traditional techniques to identify the number of latent factors: A simulation and tutorial. Psychological Methods, 25(3), 292. https://doi.org/10. 1037/met0000255

Gu, J., Strauss, C., Crane, C., Barnhofer, T., Karl, A., Cavanagh, K., \& Kuyken, W. (2016). Examining the factor structure of the 39-item and 15-item versions of the Five Facet Mindfulness Questionnaire before and after mindfulness-based cognitive therapy for people with recurrent depression. Psychological Assessment, 28(7), 791. https://doi.org/10.1037/pas0000263

Hallquist, M. N., Wright, A. G., \& Molenaar, P. C. (2019). Problems with centrality measures in psychopathology symptom networks: why network psychometrics cannot escape psychometric theory. Multivariate Behavioral Research. https://doi.org/10.1080/00273 171.2019.1640103

Heeren, A., Lannoy, S., Coussement, C., Hoebeke, Y., Verschuren, A., Blanchard, M. A., \& Gierski, F. (2021). A network approach to the five-facet model of mindfulness. Scientific Reports, 11(1), 1-11. https://doi.org/10.1038/s41598-021-94151-2

Henry, J. D., \& Crawford, J. R. (2005). The short-form version of the Depression Anxiety Stress Scales (DASS-21): Construct validity and normative data in a large non-clinical sample. British Journal of Clinical Psychology, 44(2), 227-239. https://doi.org/10.1348/ $014466505 X 29657$

Kiken, L. G., Garland, E. L., Bluth, K., Palsson, O. S., \& Gaylord, S. A. (2015). From a state to a trait: Trajectories of state mindfulness in meditation during intervention predict changes in trait 
mindfulness. Personality and Individual Differences, 81, 41-46. https://doi.org/10.1016/j.paid.2014.12.044

Kroenke, K., Strine, T. W., Spitzer, R. L., Williams, J. B., Berry, J. T., \& Mokdad, A. H. (2009). The PHQ-8 as a measure of current depression in the general population. Journal of Affective Disorders, 114(1-3), 163-173. https://doi.org/10.1016/j.jad.2008. 06.026

Medvedev, O. N., Cervin, M., Barcaccia, B., Siegert, R. J., Roemer, A., \& Krägeloh, C. U. (2021). Network analysis of mindfulness facets, affect, compassion, and distress. Mindfulness, 12(4), 911-922. https://doi.org/10.1007/s12671-020-01555-8

Medvedev, O. N., Norden, P. A., Krägeloh, C. U., \& Siegert, R. J. (2018). Investigating unique contributions of dispositional mindfulness facets to depression, anxiety, and stress in general and student populations. Mindfulness, 9(6), 1757-1767. https://doi. org/10.1007/s12671-018-0917-0

Podsakoff, P. M., MacKenzie, S. B., \& Podsakoff, N. P. (2012). Sources of method bias in social science research and recommendations on how to control it. Annual Review of Psychology, 63, 539-569. https://doi.org/10.1146/annurev-psych-120710-100452

Roca, P., Diez, G. G., Castellanos, N., \& Vazquez, C. (2019). Does mindfulness change the mind? A novel psychonectome perspective based on Network Analysis. PLoS ONE, 14(7), e0219793. https://doi.org/10.1371/journal.pone.0219793

Rohde, K., Adolph, D., Dietrich, D. E., \& Michalak, J. (2014). Mindful attention regulation and non-judgmental orientation in depression: A multi-method approach. Biological Psychology, 101, 36-43. https://doi.org/10.1016/j.biopsycho.2014.06.009
Takahashi, T., Sugiyama, F., Kikai, T., Kawashima, I., Guan, S., Oguchi, M., \& Kumano, H. (2019). Changes in depression and anxiety through mindfulness group therapy in Japan: The role of mindfulness and self-compassion as possible mediators. BioPsychoSocial Medicine, 13(1), 1-10. https://doi.org/10.1186/ s13030-019-0145-4

Truong, Q. C., Krägeloh, C. U., Siegert, R. J., Landon, J., \& Medvedev, O. N. (2020). Applying generalizability theory to differentiate between trait and state in the Five Facet Mindfulness Questionnaire (FFMQ). Mindfulness, 11(4), 953-963. https://doi.org/10. 1007/s12671-020-01324-7

Wetzels, R., \& Wagenmakers, E. J. (2012). A default Bayesian hypothesis test for correlations and partial correlations. Psychonomic Bulletin \& Review, 19(6), 1057-1064. https://doi.org/10.3758/ s13423-012-0295-x

Williams, D., \& Mulder, J. (2020). BGGM: Bayesian Gaussian graphical models in R. Journal of Open Source Software, 5(51), 2111. https://doi.org/10.21105/joss.02111

Zimmerman, M., Ellison, W., Young, D., Chelminski, I., \& Dalrymple, K. (2015). How many different ways do patients meet the diagnostic criteria for major depressive disorder? Comprehensive Psychiatry, 56, 29-34. https://doi.org/10.1016/j.comppsych.2014.09.007

Publisher's note Springer Nature remains neutral with regard to jurisdictional claims in published maps and institutional affiliations. 